\title{
FUSION OF HYPERSPECTRAL IMAGES AND LIDAR-BASED DEMS FOR COASTAL MAPPING
}

\author{
A. Elaksher \\ Cairo University, 12 abader st. alzitoon cairo, EGYPT - ahmedelaksher@yahoo.com
}

KEYWORDS: LIDAR, Hyperspectral, Rectification, Classification, Image processing.

\begin{abstract}
:
Coastal mapping is essential for a number of applications such as coastal resource management, coastal environmental protection, and coastal development and planning. Coastal mapping has been carried out using a wide range of techniques such as ground surveying and aerial mapping. Recently, satellite images, active sensor elevation models, and multispectral and hyperspectral images have also been used in coastal mapping. The integration of two or more of these datasets can provide more reliable coastal information. This paper presents an alternative technique for coastal mapping using an AVIRIS image and a LIDAR-based DEM. The DEM is used to generate building cues that are converted to building polygons. Building pixels are then removed from the AVIRIS image, and a supervised classification is performed to generate road and shoreline classes. A number of image processing techniques are used to victories road and shoreline pixels. The geometric accuracy and the completeness of the results are evaluated. The average positional accuracy for the building, road, and shoreline layers are 2.3, 5.7 and 7.2 meters, with $93.2 \%$, 91.3\%, and 95.2\% detection rates respectively. The results demonstrate the potential of using LIDAR-based DEMs to detect building cues and remove their corresponding pixels from the classification process. Thus, integrating laser and optical data can provide high quality coastal geospatial information.
\end{abstract}

\section{INTRODUCTION}

Coastal areas represent important and diverse parts of the Earth along shorelines. They consist of recreational beaches, residential regions, industrial areas, and harbors. High quality geospatial coastal information is essential for coastal resource management, coastal environmental protection, and coastal development and planning. Acquiring such information has been carried out using aerial photographs and ground surveying. These techniques have many advantages, such as flexible scheduling, easy-to-change configuration, and high quality mapping results. However, they are expensive and needs special logistics and processing procedures.

Recently, satellite-imaging systems have improved their image resolutions and opened the era for high resolution mapping from space. Pitts et al. (2004) used a series of five Landsat TM images, spanning from 1984 to 1997, for coral reefs mapping. Chang et al. (1999) used SPOT images for shoreline mapping and change detection. Drzyzga and Arbogast (2002) used IKONOS images to monitor and map coastal landscape changes during spring and late summer seasons for three sites along Lake Michigan and one site along Lake Huron. Di et al. (2003) used the multispectral IKONOS images for initial shoreline generation. The initial shoreline was then refined using the panchromatic IKONOS images.

Active remote sensing techniques, LIDAR and synthetic aperture radar (SAR), have been tested and evaluated in a wide variety of coastal applications. Cook (2003) used LIDAR for shoreline mapping and change detection in Florida coast. Tuell (1998) used SAR for shoreline mapping and change detection in a remote area along the Alaska shoreline as part of the national geodetic survey (NGS) effort to evaluate the potential of several mapping technologies. Brzank and Heipke (2006) studied the extraction of land and water areas from laser scanner datasets. Flight strips were processed separately using a fuzzy logic approach that classify the data into water and non-water classes.
The integration of active remote sensing technologies with other datasets makes it possible to provide reliable and automatic solutions for coastal mapping and change detection. Gibeaut et al. [8] used LIDAR data and historical aerial photographs to study the Gulf of Mexico shoreline changes. Lee and Shan (2003) combined LIDAR data and IKONOS images for coastal mapping. They reported an average detection rate 89.3\% without using the LIDAR data and 93\% using LIDAR data. Bartels et al. (2007) presented a rule-based approach for improving classification accuracy obtained in a supervised maximum likelihood classification process using simultaneously recorded co-registered bands such as high resolution LIDAR first, last echo and intensity data, aerial and near infra-red photos. The results show that merging these datasets improve the quality of the classification process.

This research demonstrates a new framework for automatic coastal mapping using the airborne visible/infrared imaging spectrometer (AVIRIS) and the light detection and ranging (LIDAR) systems. While AVIRIS images measure the spectral reflectance of the ground, operating with a wavelength band from $0.380 \mu \mathrm{m}$ to $2.500 \mu \mathrm{m}$ of the electromagnetic spectrum, LIDAR data are geometric range measurements, operating with a wavelength of about $1000 \mu \mathrm{m}$. Therefore, the combination of these two measurements provides accurate geometric and spectral information about the ground, which could be used to produce high quality topographic maps.

The LIDAR-based digital elevation model (DEM) and the AVIRIS image are processed and registered using a number of ground control points (GCPs) obtained from a pair of aerial images. The DEM is then filtered and segmented to generate building cues. These cues are converted to building polygons and used to generate a polygon layer for buildings. Due to the low resolution of the AVIRIS data and the diversity of the building roof materials, it is difficult to classify buildings as one class. Therefore, the building polygons are superimposed on the AVIRIS image. All buildings pixels in the AVIRIS image are 725 excluded from the classification. A supervised classification 
technique is then applied to classify the AVIRIS image. The classified pixels for the road and water classes are used to generate road and shoreline vector layers, respectively, via a line extraction process. Results showed an average positional accuracy of about five meters and an average detection rate of 93\%. The results demonstrate that integrating laser and optical data can provide high quality coastal information.

\section{DATASET DESCRIPTION}

The dataset used was collected over the coastal area of Ocean City, Maryland. Ocean City is an urban area that consists of roads, high-rise buildings, and residential buildings. In addition, along the east coast are a number of sandy beaches while harbours and docks are found on the west coast. The dataset was collected by the International Society of Photogrammetry and Remote Sensing (ISPRS) Commission III, Working Group 5, (Csathó and Schenk, 1998). The dataset includes large-scale aerial images, laser altimetry data, and hyperspectral images. The large-scale aerial photographs are used as the source for ground control points (GCPs) and as the ground truth data for evaluation. The laser altimetry and the hyperspectral data are used for the coastal mapping process.

The aerial photographs consist of a pair of 1:5000 aerial images acquired using an RC20 camera operated independently by NGS. The images are provided in a digital form at a resolution of $12.5 \mu \mathrm{m}$. Well-distributed GCPs, surveyed using the differential global positioning system (DGPS), are used to compute the interior and exterior orientation parameters of the images. A manual feature extraction process is then performed in order to provide the ground truth data.

The LIDAR-based DEM is acquired using an airborne topographic mapper (ATM) laser system. The ATM is a conical scanning laser altimeter developed by NASA for precise measurement of surface elevation changes. Laser elevation data is acquired as a point cloud that is used to drive the required DEM in three main steps. First, the flight path is reconstructed using the DGPS and the inertial navigation system (INS) techniques mounted with the ATM system on the aircraft. Secondly, the 3D coordinates for each laser pulse intersection with the ground are computed using the laser travelling time and the reconstructed flight path. The LIDAR data is generated as a high-density point cloud with an average spacing of one laser point per one square meter. Finally, a post-processing step is used for filtering out the data outliers and generating the DEM and other products. The final data is provided as a onemeter DEM projected in the universal traverse mercator (UTM) projection with the WGS84 used as the reference ellipsoid. The vertical accuracy of the LIDAR based DEM is about 10 centimetres, (Ackerman, 1999). Twenty-eight ground checkpoints, measured in the reference orthophoto, are used to evaluate the horizontal accuracy of the LIDAR based DEM. The average of the root mean square errors (RMS) for the 28 checkpoints is 1.08 meter.

The AVIRIS hyperspectral image is obtained using the AVIRIS scanner from the jet propulsion laboratory (JPL) that was installed on the NGS aircraft. The AVIRIS instrument contains 224 different detectors, each with a spectral bandwidth of approximately 10 nanometers (nm), allowing it to cover the entire range between $380 \mathrm{~nm}$ and $2500 \mathrm{~nm}$. The ground resolution of the AVIRIS image is 3.8 meters. The image is provided in raw and column pixel units with approximate geographic coordinates (latitude and longitude) for the start and the end points of the flight line. Therefore, the AVIRIS image was first registered before it is used the coastal mapping application.

\section{REGISTERING OF AVIRIS IMAGE}

Several experiments are conducted to rectify the AVIRIS image using the 2D projective transformation model, Equation 1. Three experiments are performed using different sets of GCPs and an independent set of 25 ground checkpoints. For each experiment the RMS is used to evaluate the results. The true ground coordinates of the GCPs and checkpoints are measured from the stereo images using tradition photogrammetric techniques. After computing the 2D transformation parameters using the ground and image coordinates of the GPCs, the computed parameters are used to calculate the ground coordinates of the checkpoints. These coordinates are compared with the measured ground coordinates, from the stereo images, and their RMS is computed. Results show that the average RMS is about five meters. Figure 1 shows the AVIRIS imagery before and after the rectification process using 10 GCPs.

$$
\begin{aligned}
& E=\frac{a_{1} i+a_{2} j+a_{3}}{c_{1} i+c_{2} j+1} \\
& N=\frac{b_{1} i+b_{2} j+b_{3}}{c_{1} i+c_{2} j+1}
\end{aligned}
$$

where $\quad \mathrm{E}$ and $\mathrm{N}=$ true East and North coordinates, $\mathrm{i}$ and $\mathrm{j}=$ AVIRIS imagery pixel coordinates, $\mathrm{a}_{1}, \mathrm{a}_{2}, \mathrm{a}_{3}, \mathrm{~b}_{1}, \mathrm{~b}_{2}, \mathrm{~b}_{3}, \mathrm{c}_{1}, \mathrm{c}_{2}=$ projective transformation model parameters.
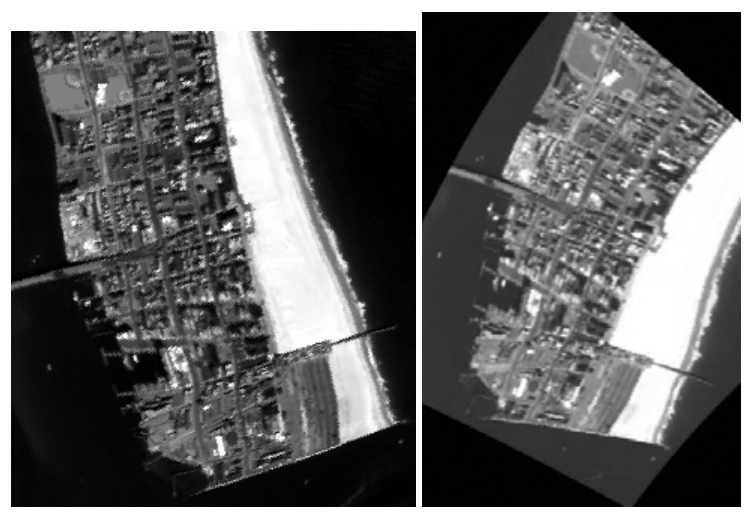

Figure 1. AVIRIS image before and after rectification using 10 GCPs

\section{COASTAL MAPPING}

The coastal mapping process is divided to two parts. In the first part, the LIDAR DEM is used to generate a vector layer for buildings. This task includes the following steps: DEM filtering, DEM segmentation, region classification, and region 
to polygon conversion. In the second part, a supervised classification technique is used to classify the AVIRIS image after removing building pixels from the image. A number of image processing techniques including thinning, edge detection, and line fitting are performed on all pixels classified as road and water to generate two vector layers for the road network and the shoreline.

\section{BUILDING VECTOR LAYER}

In order to find buildings cues in the LIDAR-based DEM, the digital surface model (DSM), i.e. representing bare ground, need to be extracted. Minimum filters are used to perform this task (Masaharu and Ohtsubo, 2002; Wack and Wimmer, 2002). The main objective of the filtering process is to detect and consequently remove points above the ground surface in order to recognize height DSM points in the data set. The minimum filter size should be large enough to include data points that are not part of the noise. However, iterative approaches could be used to avoid the effect of noise. In this research, the size of the filter is $3 \times 3$. The filtering is repeated iteratively until the DSM is extracted. If the difference between the DSM and the DEM for any pixel is greater than a given threshold, the point is treated as a building pixel. The value of the threshold is determinate using previous knowledge about the area.

The next step is to eliminate extraneous features such as trees and any other object above the ground that are not buildings. A local statistic analysis (Maas, 1999) is used for this purpose. The process is implemented as follows. Each group of pixels that lies within a small square window is fitted to a plane. Then, a least squares adjustment algorithm is used to obtain the plane parameters. After the adjustment procedure, the RMS is computed for each window. A high RMS indicates an irregular surface that can be interpreted as a characteristic of a tree or a rough surface, since most buildings have smooth roof surfaces. For each pixel, the algorithm is implemented with different window orientations and positions and the minimum RMS is reported.

A split and merge image segmentation process is then used to segment the LIDAR based DEM to regions after removing the extraneous objects from the raw data. The split and merge image segmentation technique, Horowitz and Pavlidis (1974), is implemented as follows. First a quad-tree representation is constructed for the image splitting as necessary when inhomogeneities exist. Then adjacent regions are merged to form larger regions based on a similarity criterion. In the last step, small regions are either eliminated or merged with larger regions and holes are removed. Border points for each region are extracted and used to fill a 2D Hough transformation parameter space, (Hough, 1962). The Hough transformation parameter space is then searched and analyzed to find all borderlines. For each cell in the parameter space a non-linear lest squares estimation model is employed to refine the borderline parameters using all border points contributing to the cell.

The next step is to convert the extracted lines to polygons using a rule-based system. The rules are designed as complex as possible to cover a wide range of polygons. The mechanism that is developed here works in three steps. The first step is to find all possible intersections between all borderlines. The next step is to generate all feasible polygons from all recorded intersections. Each combination of three to six intersection points is considered to be a polygon hypothesis. Some hypotheses are ignored if the difference in the area between the region and the hypothesized polygon is more than $50 \%$. The third step is to find the optimal polygon that represents the region borders. This polygon is chosen from the remaining polygons using a template matching technique. The template is chosen to be the original region, while it is matched across all polygon hypotheses. The hypothesis with the largest correlation and minimum number of vertices is chosen to be the best fitting polygon. Figure 2, shows the extracted building polygons overlaid on the one-meter LIDAR-based DEM.

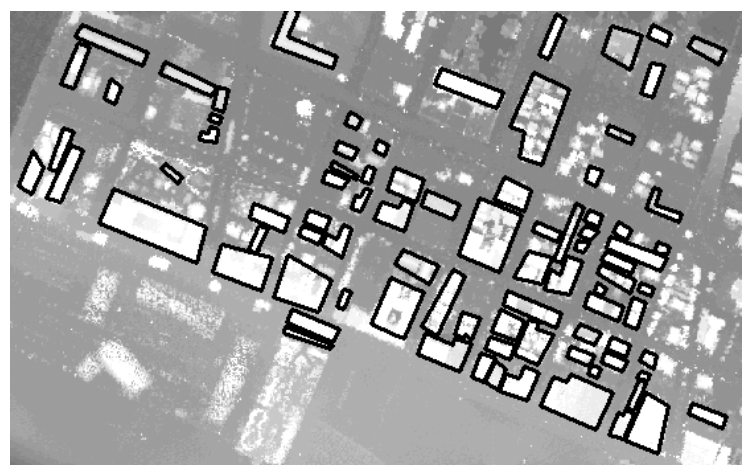

Figure 2. Extracted building polygons overlaid on the onemeter LIDAR-based DEM, (rotated)

\section{ROAD NETWORK AND SHORELINE VECTOR LAYERS}

In this section the process of generating vector layers for the road network and the shoreline is presented. The results of classifying the raw AVIRIS image showed an overall accuracy of about $68.7 \%$. This is due to the diversity of the building roof materials and the small number of buildings. Several buildings were classified as roads. This motivated the elimination of the building pixels before the classification process. Hence, the following algorithm was applied. Building polygons were first used to define building pixels in the AVIRIS hyperspectral image using a point-in-polygon process. All pixels defined as building points are identified as background pixels and not used in the classification process. In the next step, a supervised classification technique is performed on the AVIRIS hyperspectral image using the extraction and classification of homogeneous objects (ECHO) classifier, (Biehl and Landgrebe, 2002).

Six classes are defined in the image; road, water, sand beach, grass, bare soil, others. The last class includes all objects that cannot be identified. Training pixels are then identified and used to calculate each class statistics. A number of test samples are used to evaluate the classification results of each class. Table 1 shows the size of the training and test samples for each class and the classification results for the training and test samples. The total accuracy of all training sites is $92.7 \%$, while the total accuracy of all test sites is $87.6 \%$. However, the average accuracy of the road and water test sites, that are used to generate the road network and shoreline layers, is about $98 \%$. 


\begin{tabular}{|c|c|c|c|c|c|}
\hline & \multicolumn{2}{|c|}{ Number of Pixels } & \multicolumn{2}{|c|}{$\begin{array}{c}\text { Site Accuracy } \\
(\%)\end{array}$} & \multirow{2}{*}{$\begin{array}{c}\text { Number } \\
\text { of } \\
\text { Classifie } \\
\text { d Pixels } \\
\end{array}$} \\
\hline & $\begin{array}{c}\text { Trainin } \\
\text { g }\end{array}$ & Test & $\begin{array}{c}\text { Trainin } \\
\text { g }\end{array}$ & Test & \\
\hline Roads & 3686 & 1662 & 96.7 & 96.1 & 25512 \\
\hline Grass & 960 & 499 & 98.5 & 35.3 & 2047 \\
\hline Water & 3331 & 7695 & 99.6 & 99.8 & 35012 \\
\hline Sand & 2299 & 4584 & 100.0 & 99.9 & 12319 \\
\hline $\begin{array}{l}\text { Bare } \\
\text { Soil }\end{array}$ & 323 & 115 & 99.7 & 98.3 & 1312 \\
\hline Other & 7564 & 5041 & 84.1 & 59.2 & 100702 \\
\hline Total & 18163 & 19596 & 92.7 & 87.6 & 176904 \\
\hline
\end{tabular}

Table 1. Results of the AVIRIS image classification after removing the building pixels

The next step in the process is to generate the road and the shoreline vector layers. The road class is first separated from other classes then a thinning process is performed on road pixels. A 2D Hough transformation parameter space is filled using all road pixels. Peak cells in the parameter space are located and all pixels contributing to these cells are used to find the road centrelines using a non-linear lest squares estimation model. A line joining process is then used to join road centrelines that have the same parameters.

In the last step, the shoreline is extracted from the water class. First, the water class is separated from other classes and border pixels are defined. These pixels are then converted to a polyline using the algorithm presented in Bimal and Kumar (1991). The basic idea of the algorithm is to go through all the border points and only retain those that that are significant, i.e. those that represent vertices. Figure 3 shows the extracted road network and the extracted shorelines overlaid on the reference orthophoto.

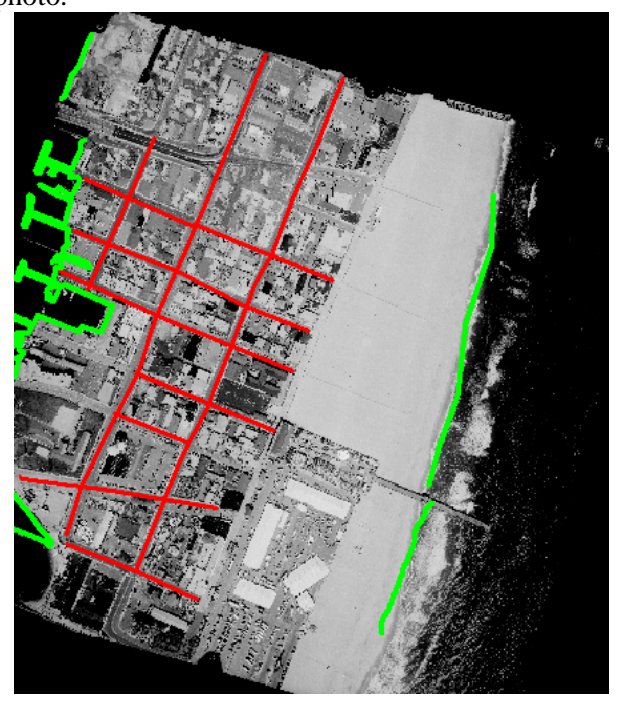

Figure 3. Extracted road network and shoreline overlaid on the reference orthophoto

\section{EVALUATION AND ANALYSIS}

The completeness and the positional accuracy of the results are evaluated using different metrics. The completeness is measured using two metrics: the detection rate (DR) and the false alarm rate (FR). Table 2 shows the values of the DR and FR for each class. The table shows that the detection rate for all three features is more than $90 \%$. In addition, the table shows that no false-alarm roads or shorelines have been extracted. The table shows that the false-alarm rate in the building layer is $3.2 \%$.

\begin{tabular}{|c|c|c|}
\hline & Detection rate (\%) & $\begin{array}{c}\text { False -alarm rate } \\
(\%)\end{array}$ \\
\cline { 1 - 1 } Roads & 91.3 & 0 \\
Shorelines & 95.2 & 0 \\
\cline { 1 - 1 } Buildings & 93.2 & 3.2 \\
\hline
\end{tabular}

Table 2. Quality metrics used to evaluate the results

The RMS is used to evaluate the positional accuracy of the extracted features. This is performed in different means. For the road network, the positions of 20 road intersections are used. The RMS for the coordinates of the 20 points is 5.7 meters and the maximum error is 9.5 meters. For the shoreline, 30 distinct points are manually selected. The RMS for the 30 points is 7.2 meters, and the maximum positional error is 10.9 meters. The RMS for the buildings corners is 2.3 meters, while the maximum error is 3.8 meters.

\section{CONCLUSIONS}

This research shows that the combination of multiple and independent remote sensing data is essential to solve the complexity of the coastal mapping. Due to the nature of the LIDAR data, it was employed to extract building polygons only. The AVIRIS data led to other coastal features that are not distinguished using the height attribute only such as roads and shorelines. The classification results are vectorized and used to generate road and shoreline vector layers. Results show that the average detection rate of the proposed technique is $93 \%$. The positional accuracy of the extracted features is data depended. The research shows that the RMS of the generated building polygons is 2.3 meters, while the RMS of the roads and the shorelines is about 6.5 meters. These results show the potential of merging optical and laser data to provide reliable and accurate geospatial information that can be used to build or update coastal GIS database.

\section{REFERENCES}

Ackerman, F., 1999. Airborne laser scanning - present status and future expectations. ISPRS Journal of Photogrammetry \& Remote Sensing, Vol. 54(2-3), pp. 64-67.

Bartels, M., Wei, H., and Ferryman, J., 2006. Analysis of LIDAR data fused with co-registered bands. Proceedings of IEEE International Conference on Advanced Video and SignalBased Surveillance, http://www.cvg.reading.ac.uk/projects/LIDAR/index.html, (accessed 6 Nov. 2007).

Biehl, L., and Landgrebe, D.A., 2002. MultiSpec: a tool for multispectral-hyperspectral image data analysis. Computers \& Geosciences, Vol. 28(10), pp. 153-1159. 
Bimal, R.K., and Kumar, S.R., 1991. A new approach to polygonal approximation. Pattern Recognition Letters, Vol. 12, pp. 229-234.

Brzank, A. and Heipke, C., 2006. Classification of LIDAR data into water and land points in coastal areas. The International Archives of Photogrammetry, Remote Sensing, and Spatial Information Sciences, Bonn, Germany, Vol. XXXV, Part I/3, pp. 197-202.

Chang, L., Chen, A.J., Chen, C.F., and Huang, C.M., 1999. A robust system for shoreline detection and it's application to coastal-zone monitoring. Proceedings of $20^{\text {th }}$ Asian Conference on Remote Sensing, (available on CD-ROM).

Cook, G., 2003. Evaluating LIDAR for documenting shoreline change. Proceedings of $3^{\text {rd }}$ Biennial Coastal GeoTools Conference, (available on CD).

Csathó, B.M., and Schenk, T., 1998. A multisensor data fusion for automatic scene interpretation, The International Archives of Photogrammetry, Remote Sensing, and Spatial Information Sciences, Columbus, Ohio, Vol. XXXII, Part 3/1, pp. 429-434.

Di, K., Wang, J., Ma, R., and Li, R., 2003. Automatic shoreline extraction from high-resolution IKONOS satellite imagery. Proceedings of 2003 ASPRS Annual Conference, (available on CD).

Drzyzga, S. and Arbogast, A., 2002. Technical notes about using IKONOS imagery to monitor and map coastal landscape change along the great lakes. Proceedings of $36^{\text {th }}$ North-Central Section and the $51^{\text {st }}$ South-eastern Section GSA Joint Annual Meeting, (available on CD).

Gibeaut, J., White, A., Hepner, T., Gutierrez, R., Tremblay, T., Smyth, R., and Andrews, J., 2007. Texas shoreline change project Gulf of Mexico shoreline change from the Brazos river to Pass Cavallo, A Report of the Texas Coastal Coordination Council pursuant to National Oceanic and Atmospheric Administration, http://www.beg.utexas.edu/coastal/report.html, ( accessed 6 Nov. 2007).

Horowitz, S.L. and Pavlidis, T., 1974. Picture segmentation by a direct split and merge procedure. Proc $2^{\text {nd }}$ International Joint Conference on Pattern Recognition, pp. 424-433.

Hough, P.V.C., 1962. Method and means for recognizing complex patterns. U. S. Patent 3,069,654.

Lee, S., and Shan, J., 2003. Combining LIDAR elevation data and IKONOS multispectral imagery for coastal classification mapping, Marine Geodesy, Vol. 26(1-2), pp. 117-127.

Maas, H.-G., 1999. The potential of height texture measures for the segmentation of airborne laser scanner data. Proceedings of $4^{\text {th }}$ Airborne Remote Sensing Conference and Exhibition, (available on CD-ROM).

Masaharu, H., and Ohtsubo, K., 2002. A filtering method of airborne laser scanner data for complex terrain, The International Archives of Photogrammetry, Remote Sensing, and Spatial Information Sciences, Vol. XXXV, Part 3B, pp. 165-169, Graz, Austria.

Pitts, R.K., Karpouzli, E., and Malthus, T.J., Remote sensing techniques for detecting change in coral reef environments: a comparative analysis. Proceedings of 2004 ASPRS Annual Conference, (available on $\mathrm{CD}$ ).

Tuell, G.H., 1998. The use of high resolution synthetic aperture radar (SAR) for shoreline mapping. The International Archives of Photogrammetry, Remote Sensing, and Spatial Information Sciences, Columbus, Ohio, Vol. XXXII, Part 3/2, pp. 592-611.

Wack, R., and Wimmer, A., 2002. Digital terrain models from airborne laser scanner data - a grid based approach, The International Archives of Photogrammetry, Remote Sensing, and Spatial Information Sciences, Vol. XXXV, Part 3B, pp. 293-296, Graz, Austria. 
